Revue internationale P.M.E.

Économie et gestion de la petite et moyenne entreprise

\title{
Lisibilité de l'environnement, management stratégique: éléments de recherche sur les PMI
}

\section{Hugues Silvestre et Régis Goujet}

Volume 9, numéro 1, 1996

URI : https://id.erudit.org/iderudit/1008254ar

DOI : https://doi.org/10.7202/1008254ar

Aller au sommaire du numéro

Éditeur(s)

Presses de l’Université du Québec

ISSN

0776-5436 (imprimé)

1918-9699 (numérique)

Découvrir la revue

Citer cet article

Silvestre, H. \& Goujet, R. (1996). Lisibilité de l'environnement, management stratégique: éléments de recherche sur les PMI. Revue internationale P.M.E., 9(1), 61-78. https://doi.org/10.7202/1008254ar

\section{Résumé de l'article}

La démarche stratégique observée dans les PMI dépend en grande partie de la turbulence qui caractérise, pour la plupart, leur contexte; turbulence qui a pour conséquence l'extrême difficulté qu'ont certains dirigeants à lire leur environnement et à y positionner leur entreprise.

Nous basant sur une étude qualitative menée auprès de 45 dirigeants de PMI, nous montrons le mode de reconstruction de l'environnement par le dirigeant à partir d'un point central constitué par son entreprise.

Nous proposons une grille d'analyse des démarches stratégiques des dirigeants de PMI en fonction, d'une part, du degré de lisibilité perçu de leur environnement et, d'autre part, de leur comportement managérial.

Enfin, nous suggérons une approche plus qualitative du concept de position à partir de la notion de rôle, ce qui nous amène à considérer l'importance, dans la démarche stratégique d'un dirigeant de PMI, de son espace de lecture ; élément dont nous précisons le sens dans le texte. 


\title{
Lisibilité de l'environnement, management stratégique: éléments de recherche sur les PMI
}

Hugues SILVESTRE

Régis GOUJET

École supérieure de commerce de Lyon

\section{MOTS CLÉS}

\section{PMI-Dirigeant-Représentation de l'environnement \\ Complexité-Turbulence-Processus stratégique \\ Positionnement stratégique}

\begin{abstract}
RÉSUMÉ
La démarche stratégique observée dans les $P M I$ dépend en grande partie de la turbulence qui caractérise, pour la plupart, leur contexte; turbulence qui a pour conséquence l'extrême difficulté qu'ont certains dirigeants à lire leur environnement et à y positionner leur entreprise.

Nous basant sur une étude qualitative menée auprès de 45 dirigeants de PMI, nous montrons le mode de reconstruction de l'environnement par le dirigeant à partir d'un point central constitué par son entreprise.
\end{abstract}

\section{LES AUTEURS}

Hugues Silvestre est responsable de recherche au Groupe école supérieure de commerce de Lyon (IRE) et il anime une équipe de recherche sur le management stratégique des PMI. II intervient en stratégie et marketing en milieu industriel et en management stratégique des PMI. II est diplômé de l'École supérieure de commerce de Paris.

Régis Goujet est chargé de recherche au Groupe école supérieure de commerce de Lyon (IRE). Ses recherches portent principalement sur le dirigeant-propriétaire d'entreprise. II intervient en management stratégique des PMI. II est titulaire d'un DEA de gestion en marketing industriel.

Adresse: Institut de recherche de l'entreprise (IRE), Axe "Management des PMI", Groupe école supérieure de commerce de Lyon, France, B.P. 174, 69132 Ecully, Cedex, France. 
Nous proposons une grille d'analyse des démarches stratégiques des dirigeants de PMI en fonction, d'une part, du degré de lisibilité perçu de leur environnement et, d'autre part, de leur comportement managérial.

Enfin, nous suggérons une approche plus qualitative du concept de position à partir de la notion de rôle, ce qui nous amène à considérer l'importance, dans la démarche stratégique d'un dirigeant de PMI, de son espace de lecture; élément dont nous précisons le sens dans le texte.

\section{ABSTRACT}

The strategic approach observed in small and medium-sized firms is greatly influenced by the turbulence which largely characterizes their context. As a consequence of this turbulence, managers have extreme difficulty in reading their environment and in positioning their firm within it.

Basing ourselves on a qualitative study carried out with 45 small and medium-sized firms managers, we demonstrate how the manager reconstructs the environment around his firm.

We present a possible analysis table of the small and medium-sized firms managers' strategic approach according to, on the one hand, the perceived degree of lisibility of their environment, and, on the other, their managerial behaviour.

Finally, we suggest a more qualitative approach to the position concept based on the notion of role, which leads us to consider the importance in the small and medium-sized firm manager's strategic approach, of his area of lisibility; the meaning of the latter is explained in the text.

\section{RESUMEN}

La gestión estratégica observada en las PyMEs depiende por parte de la turbulencia que caracterisa en varios casos su contexto. Turbulencia que tiene por consequencia una gran difficultad, por algunos responsables de las PyMEs para leer y situar sus empresas en el medio ambiente.

Gracias a una investigacion realisada cerca de 45 responsables de PyMEs, intentamos de presentar el modo de reconstrucción del medio ambiente por el responsable a partir de un punto central constituido por su empresa.

Proponemos una red de analisis de los tramites estratégicos de los responsables de PyME en función, por un lado del grado de legibicidad percibida de su entorno y por otro lado de su actitud como responsable.

Por fin, sugerimos una manera de ver mas cualitativa del concepto de posición a partir de la noción de papel. Entonces, consideramos el "espacio de lectura" del responsable como un elemento importante de la gestión estratégica de las PyMEs. 


\section{Introduction}

Le rapport entre une PMI et son environnement s'avère d'une nature particulière; quelques éléments connus illustrent cette situation : ces entreprises sont souvent plus sensibles aux aléas du marché, sans grand pouvoir pour en faire évoluer les conditions de fonctionnement. Et pourtant, elles font preuve d'une plus grande aisance à réagir avec flexibilité aux exigences des acteurs avec lesquels elles interagissent...

Ce rapport particulier est caractérisé selon nous par deux dimensions : la première est relative à la capacité plus ou moins forte de l'entreprise à lire son environnement; la seconde, à la capacité plus ou moins forte de l'entreprise à influencer son environnement.

Nous nous intéressons ici à la première dimension. Plus précisément, nous faisons l'hypothèse que le dirigeant d'une PMI - dont le rôle est considéré comme central dans la démarche stratégique de ce type d'entreprise-a un comportement stratégique largement dépendant de sa capacité à lire son environnement.

\section{Problématique}

De plus en plus nombreuses sont les PMI faisant face à un environnement plus turbulent (mondialisation des marchés, apparition de nouveaux acteurs, évolution des structures des filières, raccourcissement des cycles de vie des technologies, etc.). Dans cette situation, la problématique stratégique d'une PMI prend une dimension nettement plus complexe : elle contient des questionnements de nature identitaire. En effet, si l'environnement se caractérise par son illisibilité, l'analyse stratégique n'est plus tant un arbitrage relatif à l'allocation de ressources sur des facteurs clés, puisque les données, qui permettaient auparavant de réaliser cet exercice concrétisé par le dessin d'une trajectoire stratégique, sont devenues instables, partielles, en évolution permanente. Ainsi, lorsque la lecture précise de l'environnement leur devient inaccessible, il semble que certains dirigeants de PMI soient contraints de reconstruire celui-ci de façon partielle, le point de référence étant alors leur entreprise. Comme cette hypothèse de centralité de l'entreprise comporte des implications majeures dans la compréhension de la démarche stratégique des dirigeants de PMI, il nous a semblé nécessaire de la développer.

Nous pensons que les PMI sont nombreuses (comparativement aux grandes entreprises) à faire face à cette problématique. Dans une situation de lecture difficile de leur environnement, il est délicat pour un dirigeant de PMI de déterminer la position de son entreprise. 
Notre présent discours vise à éclaircir cette question du positionnement de la PMI selon le contexte dans lequel celle-ci se situe. Nous avons conduit une étude exploratoire qui tend à montrer que plus le dirigeant perçoit son environnement comme illisible, plus les dimensions qualitatives et la part des représentations cognitives prennent de l'importance dans la qualification de la position de son entreprise. La démarche stratégique alors mise en œuvre diffère sensiblement de la manière classique de procéder.

Ainsi, nous proposons, dans le cadre de cet article, quelques réflexions sur les points suivants.

1) Comment le dirigeant décrit-il l'environnement de son entreprise? Place-t-il son entreprise par rapport à son environnement ou dessinet-il celui-ci autour de son entreprise?

2) Comment cette prise en compte particulière de l'environnement influence l'élaboration de la stratégie et ouvre, pour l'observateur, sur un autre mode de compréhension de ce type d'entreprise?

Nous basant sur le travail terrain exploratoire de nature qualitative décrit en première partie, nous présentons et analysons les principaux résultats concernant les effets de la non-lisibilité de l'environnement sur la représentation qu'en a le dirigeant avant d'examiner, en commentaires, l'incidence du degré de lisibilité de l'environnement sur la démarche stratégique en PMI.

\section{Méthodologie}

Notre réflexion est nourrie par l'analyse et la synthèse du travail terrain que nous avons mené auprès de 45 PMI de la région Rhône-Alpes en France. Ce travail est relatif à un programme de recherche sur le management stratégique des PMI dont la phase exploratoire avait pour objectif de valider le caractère opératoire des concepts classiques de l'analyse stratégique pour ce type d'entreprise.

Une première étude concernant le concept de position-comment une PMI se décrit dans son environnement - a été réalisée auprès de 15 entreprises appartenant au secteur de la plasturgie. Toutes ces PMI sont situées dans la plastic-vallée d'Oyonnax (département de l'Ain) qui regroupe toute l'arborescence de la filière plastique avec une forte spécialisation toutefois dans la production d'outillage (moules) et de procédés de transformation du plastique à partir de la maîtrise de la technique de l'injection. Les produits finis sont les suivants : des emballages (alimentaires et flaconnage), des biens de consommation (jouets, équipement de la maison, ornements de coiffure, montures de lunettes) et des pièces techniques (automobile). Cette filière se caractérise par 
sa structure complexe, ses perspectives globalement favorables en dépit du changement radical des stratégies d'achat d'un acteur important de cette filière, à savoir la construction automobile. Les dirigeants de ces entreprises ont été interrogés à l'aide d'entretiens structurés à questions ouvertes afin de découvrir la méthode qu'ils utilisent pour déterminer la position de leur entreprise et celle des autres PMI de l'échantillon. Le traitement a consisté en une analyse croisée de leurs réponses.

Une deuxième étude a été réalisée, selon les mêmes modalités, auprès de 15 entreprises fabriquant des films et sachets plastiques situées à SainteSigolène (village d'environ 3000 habitants situé dans le département rural de la Haute-Loire). Le plateau de Sainte-Sigolène s'est spécialisé, après une reconversion à partir du tissage, dans les années 1960-1970, dans la fabrication de sachets plastiques (alimentaires, ménagers, boutiques) et de films plastiques (industriels, agricoles, bâtiment, alimentaires) par la maîtrise de deux techniques : l'extrusion et l'impression. Deux grands groupes généralistes, quelques entreprises moyennes et petites donnent à cette industrie une structure relativement concentrée située dans une filière étroite et courte, mais aux lendemains difficilement prévisibles en raison de l'évolution de la législation sur l'environnement, de la réduction du nombre des acteurs et du passage d'un marché régional/national à un marché international. Cette phase avait pour objectif d'analyser la perception des dirigeants de la position présente et future de leur entreprise dans leur filière.

Enfin, les 15 dernières entreprises ont été réunies à trois reprises en six mois. Une première fois, en amont de la phase terrain proprement dite, afin de mieux cerner les problématiques propres aux PMI lorsque leur environnement est perçu par le dirigeant comme étant turbulent. Une deuxième réunion a été organisée pendant le déroulement de l'étude de manière à interagir sur les premières informations recueillies auprès des deux premiers groupes d'entreprises. Finalement, une séance de synthèse a permis de préciser nos interprétations des conséquences sur la démarche stratégique du degré de lisibilité perçu par le dirigeant de son environnement. Ce groupe était constitué de PMI de divers secteurs aux caractéristiques disparates : équipement automobile, aéraulique, hydraulique, abrasifs, produits d'entretien, etc.

\section{Degré de lisibilité de l'environnement, problématique du positionnement de la PMI}

D'une façon classique, on admet qu'une analyse stratégique consiste, pour le stratège, à lire l'environnement et à déterminer la position que son entreprise y occupe. Puis, le comportement idéal vise à défendre, renforcer et développer cette position en fixant à l'entreprise des objectifs en adéquation avec les données 
externes. Les moyens pour atteindre ces objectifs sont inscrits dans un plan qui conditionne la trajectoire que l'entreprise doit suivre.

Le simple fait de procéder à l'exercice de positionner l'entreprise suppose que l'environnement soit lisible. Se forger une vision réaliste de son état futur sous-entend qu'il soit prévisible. Or, il semble que pour nombre de PMI ces conditions ne soient pas vérifiées. D'après la majorité des dirigeants de PMI rencontrés, c'est souvent l'environnement qui impose la trajectoire à l'entreprise et ces dirigeants réagissent à des événements que tel ou tel détecte sans les avoir prévus. Dans ces cas, la dimension stratégique des décisions consécutives à ces événements est souvent constatée a posteriori : ce sont des «stratégies réalisées », par opposition aux «stratégies intentionnelles», dirait Mintzberg (1978).

La différence entre stratégies conçues ex ante et stratégies «constatées » ex post peut être expliquée soit par le comportement stratégique du dirigeant, plus opportuniste que planificateur, soit par les caractéristiques de l'environnement de l'entreprise. Dans ce dernier cas, considérant l'entreprise comme un système sociotechnique ouvert, le dirigeant doit s'assurer de l'obtention des ressources nécessaires à la survie et à l'évolution de son entreprise. Il devra, pour ce faire, composer avec d'autres acteurs, détenteurs de ces ressources, en fonction de l'importance de celles-ci. Dès lors, le management stratégique correspond plus à une gestion de la relation avec ces détenteurs de ressources, selon le pouvoir détenu par eux et l'entreprise, qu'à l'établissement d'un plan (Pfeiffer et Salancik, 1978).

Sans parler de contrôle externe de l'organisation, la théorie de la contingence accorde à l'environnement un rôle prépondérant agissant sur les structures de l'organisation et ses fonctionnements internes (Lawrence et Lorsh, 1989). Elle établit que l'entreprise n'évolue pas dans un monde transparent et que les contingences influent sérieusement sur le pilotage de celle-ci. Nous considérons ce lien d'autant plus fort que l'environnement est caractérisé par sa turbulence; concept dont nous retenons l'approche de Joffre et Koenig (1985), à savoir : « un état de l'environnement impliquant une perte de contrôle, au moins partielle, des dirigeants sur l'avenir de leur entreprise». Cette forme d'environnement ne menace pas forcément la survie de l'entreprise, mais elle brouille les repères habituels qui permettent à une entreprise de déterminer sa position.

Dans la démarche classique, quel que soit l'état de l'environnement, l'hypothèse de lisibilité est maintenue. Le stratège responsable de la réflexion stratégique dans l'entreprise est censé occuper une situation d'observateur identifiant le système de repérage du marché, de la filière dans lequel évolue son entreprise pour placer les organisations les unes relativement aux autres avant de prendre le point de vue de son entreprise pour dessiner, à partir 
d'une position occupée et constatée ex post, une trajectoire stratégique afin d'atteindre une position visée, définie cette fois, ex ante.

Dans cette logique, la notion de position se rapproche de celle utilisée en sociologie : la place dans une hiérarchie ou un ordre stratifié. Ici, la définition de la position suppose l'existence préalable d'un système d'ordonnancement permettant le repérage d'un acteur ou d'une organisation dans son environnement ou son milieu. Ce système préalable de repérage, dans l'analyse stratégique classique, peut être assimilé à la configuration des facteurs clés de succès, configuration elle-même liée à la distance focale d'observation du marché : domaine d'activité stratégique, segment de marché, client ou groupe de clients. Une allocation de ressources sur ces facteurs clés, comparée à l'allocation faite par les concurrents, permet à l'entreprise de définir une position visée, une trajectoire. L'entreprise peut non seulement se situer, mais également se projeter et définir les moyens nécessaires pour parcourir la trajectoire prédéterminée.

Illustrons ce cas de figure en utilisant l'allégorie du jeu d'échecs. La majorité des modèles stratégiques partent de l'hypothèse implicite que le marché est comparable à une sorte d'échiquier sur lequel évoluent des acteurs dotés de plus ou moins d'atouts. À partir d'une règle du jeu commune et partagée par l'ensemble des participants - qui les maîtrisent plus ou moins en partant de leur position lisible, les acteurs décryptent la carte, évaluent les circonstances opportunes et les menaces et envisagent une trajectoire à plus ou moins long terme. L'ensemble des acteurs partage une représentation très semblable de la configuration de l'échiquier et de la place des pièces.

A contrario, considérons maintenant le cas où l'entreprise ne dispose pas d'un système de repérage «fourni » par le marché ou la filière ou susceptible d'être identifié dans ceux-ci : facteurs clés liés à la demande (dimension marché) ou facteurs liés au métier, à la technique, aux ressources amont (dimension filière). Ce manque peut être dû à différents éléments :

- l'environnement s'avère intrinsèquement difficile à lire (rupture technique, nouveaux acteurs, etc.);

- le coût d'obtention des informations est prohibitif pour la PMI considérée ;

- le système d'information de l'entreprise est défaillant;

- le dirigeant fait preuve de myopie (par exemple, il néglige les évolutions de son environnement et maintient l'application d'une recette stratégique «qui a marché » en d'autres circonstances). 
Nous avons constaté que nombreuses sont les PMI qui, pour l'une ou l'autre des raisons évoquées précédemment, ne disposent pas d'un système de repérage (tel que défini ci-dessus) préalable à leur démarche stratégique. De fait, il ressort des entretiens que nous avons conduits que lorsque la PMI est située dans un contexte instable:

- une majorité des acteurs est mal identifiée ou inconnue; seuls, les acteurs de proximité sont correctement repérés. Autrement dit, les contours de l'échiquier ne sont pas clairement établis et l'échiquier n'est pas perçu dans son ensemble;

- les perceptions de l'environnement par les différentes entreprises ne sont pas identiques (à chaque joueur correspond une image particulière de l'échiquier).

En écho à la métaphore de l'échiquier, nous en utilisons une autre pour illustrer ces situations d'illisibilité : un plaisancier en mer dans le brouillard ne disposant pas de liaison gonio/radio ne peut définir sa position à l'instant $t$ qu'en fonction d'une estimation de sa position à l'instant $t-1$. En l'absence d'amer visible, il dessine l'espace qui environne son bateau et qui devient alors son point de repère.

En effet, considérant les dirigeants de PMI que nous avons interrogés, nous avons effectivement constaté un changement de système de repérage: quand l'environnement est lisible, il est constitué de la règle du jeu de marché, mais lorsque l'environnement est considéré illisible, le dirigeant place son entreprise «au centre» et cela fait partie des éléments les plus saillants de nos observations. Plus précisément, nous avons observé qu'il ne pouvait avoir qu'une vision partielle de l'ensemble ou d'une partie des quatre éléments suivants :

- les facteurs (amont ou aval) permettant la description organisée du fonctionnement des marchés ;

- les acteurs (qu'ils soient existants, nouveaux venus ou potentiels) intervenant sur leur marché;

- la tendance (par exemple, la croissance, l'évolution technique, la concentration, etc.);

- la durée des phénomènes.

Autrement dit, dans les situations de non-lisibilité de l'environnement, il est théoriquement impossible aux dirigeants d'occuper le poste d'observateur qui leur permettrait d'avoir une vision globale de leurs environnements et des acteurs qui le composent. Lorsque nous leur avons demandé de décrire leur environnement, ils ont opéré de façon hiérarchique, classant les 
éléments du système dans lequel leur entreprise évolue selon leur proximité et leur capacité directe d'influence; leur hiérarchie s'établissait dans l'ordre suivant :

- Ceux avec qui leur entreprise est directement en interaction et qui contribuent à son évolution tels les fournisseurs, les clients, les partenaires, soit les acteurs qui conditionnent l'existence de l'entreprise et influencent l'élaboration et la mise en œuvre de la stratégie.

- Certains éléments avec lesquels les entreprises n'interagissent pas, mais que l'on ne peut tenir pour neutres, puisqu'ils contribuent largement à l'évolution du système global. Ce sont les autres entreprises du système industriel, les collectivités, etc.

- Certaines tendances résultant de l'ensemble des actions des acteurs du système qui influencent nécessairement l'évolution de l'entreprise. C'est le cas, par exemple, des innovations technologiques, des variations de conjoncture, des réorganisations de filières, etc.

Lorsqu'il n'existe pas de système de repérage, le concept de position, supposant la préexistence d'un ordre, perd son sens de même que son caractère opératoire. Nous lui préférons alors l'approche développée en marketing industriel où l'on considère que ce sont les positions des différents acteurs et les liens qui les rendent interdépendants et qui structurent les réseaux (Mattsson, 1985). Dans ce cas, la position n'est pas appréhendée à l'aide d'une fonction de repérage; elle traduit «l'importance, le rôle, le pouvoir qu'une organisation donnée exerce dans le réseau, c'est-à-dire sa capacité à influencer les autres acteurs avec lesquels elle est en relation» (Salle et Silvestre, 1992).

Autre constat de notre étude : placé dans un environnement illisible, le dirigeant est surtout sensible à certains événements qu'il interprète en fonction de leur influence sur son entreprise. En fait, il raisonne plus en termes de contexte que d'environnement. Le concept de contexte que nous utilisons ici a pour contenu celui proposé par Håkansson et Snehota (1990). Ces auteurs utilisent une approche réseau pour analyser l'interface environnementorganisation dans le cadre de la démarche stratégique d'une entreprise. Ils notent la forte interdépendance des acteurs en milieu industriel et considèrent nécessaire de prendre en compte l'interaction entre acteurs dans l'analyse de l'environnement. Aussi préfèrent-ils substituer au concept d'environnement celui de contexte constitué des interactions ayant lieu entre une entreprise et d'autres acteurs.

De fait, nous avons observé que les dirigeants retiennent tel ou tel événement issu de l'action de tel ou tel acteur avec lequel leur entreprise est 
en interaction plus ou moins intense. Cela revient à privilégier la lecture de l'environnement en termes d'acteurs plutôt qu'en termes de facteurs.

\section{Commentaires}

\subsection{Importance des représentations du dirigeant dans la démarche stratégique}

Nous avons remarqué, parmi les PMI analysées, que la principale conséquence de l'absence de système de repérage fourni par le marché était une reconstruction de l'environnement à partir d'elles-mêmes. L'environnement n'est pas une donnée, mais un construit (Weick, 1969). Les dirigeants considèrent plus le contexte dans lequel elles se trouvent que l'environnement, l'impact de l'événement prend le pas sur la tendance; ils focalisent sur certains acteurs ou certains faits plutôt que sur l'ensemble de leur marché. La subjectivité de la démarche se renforce.

En conséquence, il est primordial de prendre en compte, non pas l'environnement appréhendé de façon quasi objective, comme pourrait le faire un observateur extérieur, mais plutôt la représentation mentale que se fait le dirigeant de la réalité qui l'entoure.

Nous retenons comme définition de la représentation, l'activité mentale du dirigeant qui consiste à organiser sa connaissance de la réalité. Il s'agit donc, à partir de la perception de certains signaux de l'environnement, d'un phénomène de reconstruction de la réalité. «Elle n'est pas un simple décalque, elle sélectionne et distord, en fonction de la position qu'occupent les individus dans une situation sociale donnée et des relations qu'ils ont avec autrui.» (Fisher, 1987)

Nous pouvons à ce stade émettre l'hypothèse que l'absence de repérage renforce l'importance du dirigeant et de ses caractéristiques personnelles dans la construction de la représentation de son environnement. Cela nous conduit à considérer plus particulièrement le mode de prise de décision stratégique d'un dirigeant de PMI, mode dont nous retenons ici quelques éléments.

On constate en effet que des PMI aux caractéristiques très proches (secteurs d'activité et géographique, taille, outil de production, etc.) à un instant $t$ peuvent suivre des évolutions fort différentes par la suite, et ce, en grande partie du fait des caractéristiques du dirigeant. Celui-ci traite des informations, matériaux à la prise de décision. Puis, en fonction de ces informations, d'un ensemble d'attitudes et de sa vision, il façonne et cherche à mettre en œuvre des actions, les outputs, qui orientent l'évolution de l'entreprise. 
Cela correspond à la détermination plus ou moins rationnelle d'une stratégie qui engage l'entreprise dans l'avenir.

Par exemple, il est fort possible qu'un événement imprévu ou qu'une prise de conscience particulière soient perçus de façon très exacerbée. Cela précipitera une décision parfois à caractère stratégique, uniquement focalisée sur cette perception de «l'information». Certaines PMI sont dans l'obligation d'orienter leurs choix stratégiques sous la pression de la nécessité, dans des conditions d'ambiguïté, les objectifs étant rendus vagues en raison de l'extrême turbulence de l'environnement.

Dès lors, la conséquence de l'absence de système de repérage sur la démarche stratégique peut être analysée selon deux dimensions :

- le processus de prise en compte de tel événement plutôt que tel autre et la gestion subséquente de cette information sous la forme d'une décision stratégique. On s'oriente ici vers les travaux de compréhension de la formation de l'agenda stratégique du dirigeant (Laroche, 1991);

- le processus de décision du dirigeant, processus dans lequel les caractéristiques cognitives du dirigeant ont une forte incidence (Cossette, 1993).

Sur ce dernier point, dans le cas d'illisibilité perçue, la comparaison des options stratégiques après inventaire quasi exhaustif (comme supposé dans la démarche classique) n'est plus possible. Le dirigeant considère donc ces options, comme elles se présentent, de façon séquentielle. La démarche stratégique, dans ce cas, correspond plus à des décisions stratégiques dont il s'agit de mieux comprendre le processus (Julien et Marchesnay, 1991).

\subsection{Types d'environnements et types de comportements stratégiques}

À ce stade de la réflexion, ayant constaté l'incidence du degré de lisibilité de l'environnement sur le positionnement de la PMI et le rôle central des représentations du dirigeant, nous proposons une grille synthétique dans laquelle sont identifiés différents comportements stratégiques de dirigeants de PMI. Le comportement du dirigeant dépend de ses caractéristiques et de celles de l'environnement.

Parmi les diverses typologies de dirigeants de PMI, on retiendra pour sa clarté et sa pertinence celle de Julien et Marchesnay (1987) qui met en évidence deux types opposés de dirigeants sur l'évolution de leur entreprise : les entrepreneurs «PIC » et «CAP». Les premiers optent pour des actions privilégiant la pérennité de leur entreprise alors que les seconds recherchent en 
priorité la croissance. Au sein de ces motivations, on constate bien sûr des compromis entre ces deux classes de dirigeants. Compromis qui résultent de l'existence et de l'importance de différentes variables. On citera, à titre d'exemple, le nombre de décisionnaires et la répartition des rôles, l'arbitrage risque / pouvoir relatif au problème de «l'argent frais » (ouverture ou non du capital), la logique de la raison d'être (capitalistique, industrielle, sociale, affective, etc.), les caractéristiques objectives du dirigeant (études, expérience, âge, etc.) ou bien subjectives, notamment ses valeurs dont Bamberger (1982) précise qu'il y a une relation étroite entre celles-ci et les objectifs poursuivis par les dirigeants de PMI.

Du croisement de la lisibilité de l'environnement et des caractéristiques du dirigeant émergent des comportements illustrés par le tableau suivant dans lequel on place les deux types de dirigeants CAP et PIC dans la situation où ils disposent ou non d'un système de repérage de leur environnement.

TABlEAU 1

Comportements stratégiques du dirigeant

\begin{tabular}{llll}
\hline & & \multicolumn{2}{c}{ Caractéristiques de l'environnement } \\
\cline { 2 - 4 } & Repérage & $\begin{array}{l}\text { Non } \\
\text { repérage } \\
\text { Options } \\
\text { stratégiques } \\
\text { traitées de } \\
\text { façon } \\
\text { séquentielle }\end{array}$ \\
\cline { 2 - 4 } $\begin{array}{l}\text { Type de } \\
\text { dirigeant }\end{array}$ & $\begin{array}{l}\text { Options } \\
\text { stratégiques } \\
\text { traitées par } \\
\text { comparaison }\end{array}$ & Proactif & Opportuniste \\
\cline { 2 - 4 } & PIC & Réactif & Attentiste \\
\hline
\end{tabular}

Bien que schématique, cette grille permet d'illustrer quatre types de comportements stratégiques.

- Proactif et réactif dans un environnement repérable autorisant l'application de l'analyse stratégique classique et de la définition d'une position visée en fonction d'une « règle du jeu » du marché. Le comportement proactif correspond à un dirigeant qui, connaissant la règle du jeu de marché, investit en prévision des évolutions de celui-ci; le comportement réactif étant le propre du dirigeant

- qui investirait après constatation des mêmes évolutions. 
- Opportuniste et attentiste dans un environnement sans système de repérage. Le comportement que nous qualifions d'opportuniste est typique du dirigeant qui «saisit» telle ou telle occasion alors que le dirigeant au comportement attentiste attendrait des conditions permettant une prise de décision moins risquée, dans un contexte plus «certain».

Cette représentation cardinale des comportements stratégiques des dirigeants est structurée sur la base de deux dimensions, l'une caractéristique de l'environnement, l'autre des types de dirigeant. Elles sont dans la réalité bien plus hétérogènes. Ainsi, sur la première dimension, nous avons pu observer que la lisibilité prise en compte est plus une lisibilité perçue qu'une lisibilité objective.

Si l'on retient la distinction entre lisibilité perçue et lisibilité objective, cela nous permet d'analyser la pertinence méthodologique de la démarche stratégique (tableau 2); pertinence qui dépend de la cohérence entre le caractère lisible et illisible de l'environnement (ce qui correspond au fait que soit accessible ou non un système de repérage issu du marché) et la lisibilité telle qu'elle est perçue par le dirigeant.

TABLEAU 2

Comportements et démarches stratégiques

\begin{tabular}{cccc}
\hline Environnement & $\begin{array}{c}\text { Repérage } \\
\text { possible }\end{array}$ & $\begin{array}{c}\text { Repérage } \\
\text { impossible }\end{array}$ & $\begin{array}{c}\text { Comportement } \\
\text { stratégique } \\
\text { observé }\end{array}$ \\
\hline $\begin{array}{c}\text { Environnement } \\
\text { perçu comme lisible }\end{array}$ & $\begin{array}{c}\text { La comparaison } \\
\text { classique des } \\
\text { options stratégiques } \\
\text { est possible. }\end{array}$ & $\begin{array}{c}\text { La comparaison } \\
\text { classique des options } \\
\text { stratégiques est } \\
\text { erronée. }\end{array}$ & Proactif \\
\hline $\begin{array}{c}\text { Environnement } \\
\text { perçu comme } \\
\text { illisible }\end{array}$ & $\begin{array}{c}\text { L'approche } \\
\text { séquentielle est } \\
\text { réductrice dans } \\
\text { l'analyse stratégique. }\end{array}$ & $\begin{array}{c}\text { Les options } \\
\text { stratégiques sont à } \\
\text { juste titre considérées } \\
\text { de façon séquentielle. }\end{array}$ & Opportuniste \\
\hline
\end{tabular}

On constate que la planification stratégique n'est pertinente d'un point de vue méthodologique et autorisée par l'environnement que dans l'un des quatre cas : au croisement de «repérage possible» et «environnement perçu comme lisible». Cela alimente le débat sur la planification stratégique en PMI (Robinson et Pearce, 1988; d'Amboise et Bakanibona, 1990). 


\subsection{Synthèse-lisibilité et démarche stratégique}

En résumé, la turbulence pose un réel problème aux dirigeants de PMI qui, dans le cadre de leur démarche stratégique, désirent établir la position de leur entreprise et la gérer: les hypothèses de l'approche stratégique classique, linéarité, globalité et lisibilité ne sont pas remplies. Bien souvent, le dirigeant est «assujetti » à considérer les options stratégiques de façon séquentielle et à faire le choix de telle ou telle option sans pouvoir la comparer simultanément avec d'autres.

Dans ces conditions, les compétences internes de l'entreprise prennent une importance déterminante. Le point de départ de la réflexion stratégique est l'entreprise qui cherche un terrain d'expression correspondant à ses compétences. À partir de cette constatation, nous orientons nos recherches vers une réflexion et le développement d'outils d'analyse et de construction de l'identité d'une PMI. Ce dernier concept est à entendre ici selon la définition de Mattsson (1985): «strategic identity», qui considère l'interaction entre l'entreprise et les acteurs du réseau dans lequel elle s'inscrit comme descriptive de son rôle dans ce réseau et de son identité.

Nous reprenons en synthèse le parallèle entre une démarche classique appropriée en cas de lisibilité perçue et l'émergence d'une autre démarche (exploitation des compétences internes) liée à une hypothèse d'illisibilité perçue. Nous retenons pour cette comparaison cinq éléments de la démarche stratégique croisés avec la perception de la lisibilité de l'environnement par le dirigeant de la PMI. On constate à la lecture du tableau suivant (tableau 3) que le contenu de la démarche adoptée varie selon que le dirigeant perçoit ou non son environnement comme lisible.

Le système de repérage, c'est-à-dire les éléments auxquels le dirigeant peut se référer pour décrire la position de sa PMI dans l'environnement. Lorsque ce dernier est lisible, le dirigeant connaissant les éléments mesure les effets de la présence de son entreprise sur tel ou tel facteur clé de succès. Mais si l'environnement est considéré illisible, il décrypte les acteurs de son environnement, en partant cette fois, de son entreprise. L'environnement est reconstruit à partir de son entreprise alors que, dans le cas inverse, l'environnement étant lisible, les facteurs clés de succès lui sont donnés. Ainsi, la détermination de la position peut se réaliser de façon quantitative lorsque l'environnement est lisible, les parts de marché et la présence sur les facteurs clés sont mesurables. Inversement, la mesure de la position devient largement qualitative si l'environnement est perçu comme illisible, le dirigeant appréciant alors le rôle tenu par son entreprise auprès des acteurs qu'il considère stratégiques pour son évolution. 


\section{TABleau 3}

\section{Lisibilité et démarche stratégique}

Lisibilité perçue par le dirigeant de PMI

\begin{tabular}{ccc}
\hline $\begin{array}{c}\text { Dimensions de l'analyse } \\
\text { stratégique }\end{array}$ & $\begin{array}{c}\text { Lisibilité } \\
\text { perçue }\end{array}$ & $\begin{array}{c}\text { Illisibilité } \\
\text { perçue }\end{array}$ \\
\hline Système de repérage & Facteurs clés de succès. & $\begin{array}{c}\text { Compétences internes et } \\
\text { centralité de l'entreprise. }\end{array}$ \\
\hline $\begin{array}{c}\text { Mode d'appréhension } \\
\text { de l'environnement }\end{array}$ & $\begin{array}{c}\text { L'environnement } \\
\text { est donné. }\end{array}$ & $\begin{array}{c}\text { L'environnement est construit } \\
\text { par le dirigeant. }\end{array}$ \\
\hline $\begin{array}{c}\text { Mode de } \\
\text { positionnement }\end{array}$ & $\begin{array}{c}\text { Position appréciée de façon } \\
\text { relative en termes de performance. }\end{array}$ & $\begin{array}{c}\text { Position qualitative } \\
\text { appréciée à l'aide } \\
\text { de la notion de rôle. }\end{array}$ \\
\hline Méthode de la & $\begin{array}{c}\text { Allocation de ressources } \\
\text { sur les facteurs clés. } \\
\text { Planification et } \\
\text { Prospective }\end{array}$ & $\begin{array}{c}\text { Gestion des acteurs et } \\
\text { opportunités par proximité. } \\
\text { Vision d'un état } \\
\text { identitaire futur. }\end{array}$ \\
\hline
\end{tabular}

Dans cette situation, le dirigeant gère plutôt des acteurs et des occasions d'affaires qu'il décèle en fonction de leur proximité, se basant pour les juger, d'une part, sur les compétences internes de son entreprise et, d'autre part, sur la vision qu'il a de l'identité future de son entreprise. Cette dernière notion traduit les compétences propres et le mode de fonctionnement que l'entreprise doit acquérir ou développer pour accroître la satisfaction que son dirigeant a du rôle de son entreprise dans son environnement. La proximité liée à une action stratégique se mesure alors en fonction de l'adéquation de la décision avec la vision que le dirigeant a de l'évolution de l'entreprise. Par opposition, dans le cas de lisibilité, l'élaboration d'un plan stratégique, donc d'une trajectoire précisée est permise, la démarche contient alors la problématique du choix de l'allocation de ressources sur tel ou tel facteur clé.

Les deux modèles synthétisés dans le tableau présentent des comportements induits diamétralement opposés. Il nous paraît important de souligner qu'au sein d'une même PMI conduite par un dirigeant unique, ce dernier peut adopter les deux comportements.

- Ce peut être le cas, par exemple, d'un dirigeant de PMI ayant plusieurs domaines d'activités. Certains de ces domaines peuvent être perçus par le dirigeant comme illisibles, auquel cas ce dernier adopte un comportement en conséquence, et par ailleurs, au même moment, il perçoit l'environnement d'une autre activité comme très lisible, privilégiant alors un comportement stratégique plus classique. 
- Cela peut aussi, dans le cas d'une PMI mono-activité, résulter de l'angle d'analyse adopté par le dirigeant. Prenons l'exemple de la plasturgie qui montre que la perception de la lisibilité dépend de l'envergure de l'environnement considéré par le dirigeant.

- Les transformateurs ont une vision assez limpide de leur domaine d'activité pour lesquels les évolutions techniques sont connues et les perspectives d'évolution identifiées.

- Par contre, les segments de marchés se révèlent peu lisibles, principalement en raison des réorientations des stratégies des grands donneurs d'ordres pour faire face à une concurrence internationale accrue. Les stratégies d'achat changent ( $c f$. la construction automobile) et les applications de l'évolution technique liées au plastique sont floues.

- Lorsque la lisibilité de tel ou tel client est bonne. Effectivement, les transformateurs se concentrent sur quelques clients clés avec lesquels ils entretiennent des relations stables permettant des développements technologie/ application dans le temps.

\section{Conclusion}

À partir de notre étude conduite auprès de 45 PMI, nous avons souligné l'incidence de la lisibilité perçue sur la démarche stratégique d'un dirigeant de PMI. En particulier, nous avons constaté le manque d'opérationnalité du concept classique de position dans la démarche stratégique en PMI. C'est pourquoi, lorsque le dirigeant perçoit de l'illisibilité dans l'environnement de son entreprise, nous préférons substituer au concept de position un concept composite qui, pour un acteur donné, dans un contexte donné, est constitué des trois dimensions interdépendantes suivantes : la position appréhendée de façon qualitative, c'est-à-dire le rôle ou la représentation qu'en a le dirigeant et les actions qui en découlent (figure 1).

FIGURE 1

REPRÉSENTATION

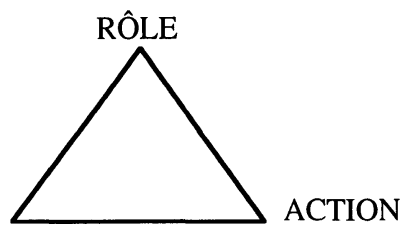


Dans le cas d'un environnement perçu comme étant non lisible, nous pensons que le dirigeant recherche un espace de lecture au sein duquel il peut à la fois lire son entreprise, la décrire et la prévoir, c'est-à-dire se la représenter dans un état identitaire futur.

On peut alors émettre l'hypothèse que, dans leur démarche stratégique, les dirigeants de PMI recherchent un espace de lecture tel qu'ils puissent avoir alors, au sein de cet espace, une représentation du rôle de leur entreprise et des actions susceptibles de légitimer ce rôle.

\section{Bibliographie}

BAmberger, I. (1982), «Les valeurs de dirigeants des PME et leur influence sur le comportement stratégique et la performance de leurs entreprises », Économies et Sociétés, série Sciences de Gestion, décembre, p. 1353-1383.

CossetTe, P. (1993), «La vision stratégique du propriétaire-dirigeant de PME : une étude cartographie cognitive », Communication présentée au Congrès international francophone de la PME, GREPME, 28-30 octobre, Carthage.

D’AMBoise, G. (1986), «Gestion stratégique des PME : les modèles émergents de la littérature », Document de travail nº 86-31, Université Laval, Québec, 23 p.

D’ Amboise, G. et A. BAKAniBona (1990), «La planification dans les PME: une synthèse de résultats empiriques. Conclusions et recommandations », Revue Internationale PME, vol. 3, $\mathrm{n}^{0}$ 2, p. 147-166.

FISHER, G.N. (1987), Les concepts fondamentaux de la psychologie sociale, Montréal, Presses de l'Université de Montréal ; Paris, Dunod, p. 208.

HÅKANSSON, H. et I. SNEHOTA (1990), "No business in an island: the network concept of business strategy », dans D. Ford (dir.), Understanding Business Market, Londres, Les Presses de l'Académie, p. 527-540.

Joffre, P. et G. KoENIG (1985), «L'évolution de la pensée stratégique », Revue Française de Gestion, mars-avril-mai, p. 68-86.

Julien, P.A. et M. MARChESnay (1987), La petite entreprise, Paris, Vuibert, p. 280.

JULIEN, P.A. et M. MARCHESNAY (1991), « Des procédures aux processus stratégiques dans les $P M E$ », Québec, Université du Québec à Trois-Rivières, Cahiers de recherche du GREPME, no 91-14, p. 29.

LAROCHE, H. (1991), La formulation des problèmes stratégiques. Agenda stratégique et identité de l'entreprise, Thèse de doctorat, Montréal, HEC, p. 451.

LAWRENCE, P. et J.W. LORSH (1989), Adapter les structures de l'entreprise : intégration ou différenciation, Paris, Éditions d'Organisation, p. 237.

Martinet, A.C. (1983), Stratégie, Paris, Vuibert, p. 322. 
MATTSSON, L.G. (1985), "An application of network approach to marketing: defending and changing market position », dans N. Dholakia et J. Arndt (éd.), Changing the Course of Marketing: Alternative Paradigms for Widening Marketing Theory, Research in Marketing, Greenwich, JAI Press.

MiNTZBER, G.H. et al. (1976), "The structure of unstructured decision process », Administrative Science Quarterly, vol. 21, p. 246-275.

MintzBer, G.H. (1978), «Patterns in strategy formation», Management Science, p. 934-948, cité par I. BAMBERGER (1982).

Pfeiffer, J. et G.R. SAlanciK (1978), The External Control of Organizations. A Resource Depence Perspective, New York, Harper et Row, p. 301.

Robinson, R.B. et J.A. PEARCE (1988), « Planned patterns of strategic behavior and their relationship to business-unit performance », Strategic Management Journal, vol. 9 , p. 43-60.

SAlle, S. et H. Silvestre (1992), Vendre à l'industrie; approche stratégique de la relation business to business, Paris, Éditions Liaisons, p. 135.

WEICK, K.E. (1979), The Social Psychology of Organizing, $2^{\mathrm{e}}$ édition, New York, McGraw-Hill, p. 294. 